\title{
Deformations of Pre-Symplectic Structures: a Dirac Geometry Approach
}

\author{
Florian SCHÄTZ $Z^{\dagger}$ and Marco ZAMBON ${ }^{\ddagger}$ \\ † University of Luxembourg, Mathematics Research Unit, Maison du Nombre 6, \\ avenue de la Fonte L-4364 Esch-sur-Alzette, Luxembourg \\ E-mail: florian.schaetz@gmail.com \\ $\ddagger$ KU Leuven, Department of Mathematics, Celestijnenlaan 200B box 2400, \\ BE-3001 Leuven, Belgium \\ E-mail: marco.zambon@kuleuven.be
}

Received September 24, 2018, in final form November 27, 2018; Published online December 06, 2018 https://doi.org/10.3842/SIGMA.2018.128

\begin{abstract}
We explain the geometric origin of the $L_{\infty}$-algebra controlling deformations of pre-symplectic structures.
\end{abstract}

Key words: pre-symplectic geometry; deformation theory; Dirac geometry

2010 Mathematics Subject Classification: 17B70; 53D17; 58H15

\section{Introduction}

A pre-symplectic form is just a closed 2-form of constant rank. For instance, the restriction of a symplectic form to a coisotropic submanifold (such as the zero level set of a moment map) is pre-symplectic. Given a pre-symplectic from $\eta$ of rank $k$, we constructed in [7] an algebraic structure that encodes the deformations of $\eta$, i.e., the 2 -forms nearby $\eta$ (in the $C^{0}$-sense) which are both closed and of constant rank $k$. As in many deformation problems, this algebraic structure is an $L_{\infty}$-algebra, which we call the Koszul $L_{\infty}$-algebra of $\eta$. Its construction - which is somewhat involved due to the simultaneous presence of the closedness and constant rank conditions - relies on a certain $B V_{\infty}$-algebra structure on the differential forms and builds on the work of Fiorenza-Manetti [1]. The Koszul $L_{\infty}$-algebra has the property that its MaurerCartan elements are in bijection with the pre-symplectic deformations of $\eta$.

Given that pre-symplectic forms are geometric objects, it is natural to ask for a geometric derivation of the algebraic structure that governs their deformations (the Koszul $L_{\infty}$-algebra). The present note provides an answer to this question. The idea is the following: instead of restricting oneself to the realm of 2-forms, work in the larger class of almost Dirac structures, and consider deformations of

$$
\operatorname{graph}(\eta):=\{(v, \eta(v, \cdot)) \mid v \in T M\} \subset T M \oplus T^{*} M
$$

within the Dirac structures satisfying a constant rank condition. This is explained in Section 3.2, which is the heart of this note.

The first step in [7] is to provide a parametrization of the constant rank forms nearby $\eta$ in terms of (an open subset in) a vector space. This parametrization is obtained naturally by taking the point of view of Dirac linear algebra in Section 3.3.

The second step in [7] was to show that the closedness condition translates into a MaurerCartan equation for a suitable $L_{\infty}$-algebra. In Section 3.4 we re-obtain this result, and further we improve slightly a result of [7], see our Corollary 2.9 . 
Combining these results, in Section 3.5 we recover the fact that the $L_{\infty}$-algebra governing deformations of Dirac structures, in the case at hand and upon a suitable restriction, is the Koszul $L_{\infty}$-algebra.

The Koszul $L_{\infty}$-algebra depends on an auxiliary choice of a distribution transverse to $\operatorname{ker}(\eta)$. In the Dirac-geometric interpretation, this translates into a suitable choice of a complement of $\operatorname{graph}(\eta)$ in $T M \oplus T^{*} M$. One of the achievements of [3] is to establish a general framework to control the effects of changing the complement, exhibiting explicit canonical $L_{\infty}$-isomorphisms between the corresponding $L_{\infty}$-algebras. A consequence of this note and of [3] is that the Koszul $L_{\infty}$-algebra of $(M, \eta)$ is well-defined up to $L_{\infty}$-isomorphisms.

\section{Review: deformations of pre-symplectic structures}

We review the results on deformations of pre-symplectic structures obtained in the first three sections of [7].

\subsection{Pre-symplectic structures}

Fix a smooth manifold $M$.

Definition 2.1. A 2 -form $\eta$ on $M$ is called pre-symplectic if

1) $\eta$ is closed,

2) the vector bundle map $\eta^{\sharp}: T M \rightarrow T^{*} M, v \mapsto \iota_{v} \eta=\eta(v, \cdot)$ has constant rank.

A pre-symplectic manifold is a pair $(M, \eta)$ consisting of a manifold $M$ and a pre-symplectic structure $\eta$ on $M$. We denote the space of all pre-symplectic structures of rank $k$ on $M$ by $\operatorname{Pre}^{-S y m}{ }^{k}(M)$.

A pre-symplectic manifold $(M, \eta)$ gives rise to a distribution

$$
K:=\operatorname{ker}\left(\eta^{\sharp}\right) .
$$

This distribution is involutive since $\eta$ is closed, hence $K$ is tangent to a foliation of $M$. Denote by $r: \Omega(M) \rightarrow \Gamma\left(\wedge K^{*}\right)$ the restriction map. We define the horizontal differential forms as the elements of

$$
\Omega_{\mathrm{hor}}(M):=\operatorname{ker}(r)
$$

They form a subcomplex of the de Rham complex $\Omega(M)$, since the de Rham differential commutes with the pullback of differential forms. The subcomplex $\Omega_{\text {hor }}(M)$ is the multiplicative ideal of $\Omega(M)$ generated by $\Gamma\left(K^{\circ}\right)$, where $K^{\circ} \subset T^{*} M$ denotes the annihilator of $K$.

\subsection{A parametrization of constant rank 2-forms}

In this subsection we fix a finite-dimensional, real vector space $V$. Recall that a bivector $Z \in \wedge^{2} V$ is encoded by the induced linear map

$$
Z^{\sharp}: \quad V^{*} \rightarrow V, \quad \xi \mapsto \iota_{\xi} Z=Z(\xi, \cdot) .
$$

Define

$$
\mathcal{I}_{Z}:=\left\{\beta \in \wedge^{2} V^{*}: \operatorname{id}_{V}+Z^{\sharp} \beta^{\sharp} \text { is invertible }\right\},
$$


an open neighborhood of the origin in $\wedge^{2} V^{*}$. Let $F: \mathcal{I}_{Z} \rightarrow \wedge^{2} V^{*}$ be the map determined by

$$
(F(\beta))^{\sharp}=\beta^{\sharp}\left(\mathrm{id}+Z^{\sharp} \beta^{\sharp}\right)^{-1} .
$$

The map $F$ is non-linear and smooth. It is a diffeomorphism from $\mathcal{I}_{Z}$ to $\mathcal{I}_{-Z}$, which keeps the origin fixed.

Fix $\eta \in \wedge^{2} V^{*}$ of rank $k$. We now use $F$ to construct submanifold charts for the space $\left(\wedge^{2} V^{*}\right)_{k}$ of skew-symmetric bilinear forms on $V$ of $\operatorname{rank} k$. Fix a subspace $G \subset V$ such that $K \oplus G=V$, where $K=\operatorname{ker}\left(\eta^{\sharp}\right)$. The restriction of $\eta$ to $G$ is a non-degenerate skew bilinear form, therefore there is a unique $Z \in \wedge^{2} G \subset \wedge^{2} V$ such that

$$
Z^{\sharp}: \quad G^{*} \rightarrow G, \quad \xi \mapsto \iota_{\xi} Z=Z(\xi, \cdot)
$$

equals $-\left(\left.\eta\right|_{G} ^{\sharp}\right)^{-1}$.

Definition 2.2. The Dirac exponential map $\exp _{\eta}$ of $\eta$ (and for fixed $G$ ) is the mapping

$$
\exp _{\eta}: \mathcal{I}_{Z} \rightarrow \wedge^{2} V^{*}, \quad \beta \mapsto \eta+F(\beta)
$$

Let $r: \wedge^{2} V^{*} \rightarrow \wedge^{2} K^{*}$ be the restriction map; we have the natural identification $\operatorname{ker}(r) \cong$ $\wedge^{2} G^{*} \oplus\left(G^{*} \otimes K^{*}\right)$. By the following theorem [7, Theorem 2.6], the restriction of $\exp _{\eta}$ to $\operatorname{ker}(r)$ is a submanifold chart for $\left(\wedge^{2} V^{*}\right)_{k} \subset \wedge^{2} V^{*}$.

Theorem 2.3 (parametrizing constant rank forms).

(i) Let $\beta \in \mathcal{I}_{Z}$. Then $\exp _{\eta}(\beta)$ lies in $\left(\wedge^{2} V^{*}\right)_{k}$ if, and only if, $\beta$ lies in $\operatorname{ker}(r)=\left(K^{*} \otimes G^{*}\right) \oplus$ $\wedge^{2} G^{*}$.

(ii) Let $\beta=(\mu, \sigma) \in \mathcal{I}_{Z} \cap\left(\left(K^{*} \otimes G^{*}\right) \oplus \wedge^{2} G^{*}\right)$. Then $\exp _{\eta}(\beta)$ is the unique skew-symmetric bilinear form on $V$ with the following properties:

- its restriction to $G$ equals $\left.(\eta+F(\sigma))\right|_{\wedge^{2} G}$;

- its kernel is the graph of the map $Z^{\sharp} \mu^{\sharp}=-\left(\left.\eta\right|_{G} ^{\sharp}\right)^{-1} \mu^{\sharp}: K \rightarrow G$.

(iii) The Dirac exponential map $\exp _{\eta}: \mathcal{I}_{Z} \rightarrow \wedge^{2} V^{*}$ restricts to a diffeomorphism

$$
\mathcal{I}_{Z} \cap\left(\left(K^{*} \otimes G^{*}\right) \oplus \wedge^{2} G^{*}\right) \stackrel{\cong}{\longrightarrow}\left\{\eta^{\prime} \in\left(\wedge^{2} V^{*}\right)_{k} \mid \text { ker }\left(\left(\eta^{\prime}\right)^{\sharp}\right) \text { is transverse to } G\right\}
$$

onto an open neighborhood of $\eta$ in $\left(\wedge^{2} V^{*}\right)_{k}$.

Remark 2.4. By the above linear algebra construction, given a pre-symplectic manifold $(M, \eta)$, choosing a subbundle $G$ complementary to $K=\operatorname{ker}\left(\eta^{\sharp}\right)$, one obtains a map

$$
\exp _{\eta}: \mathcal{I}_{Z} \cap\left(\left(K^{*} \otimes G^{*}\right) \oplus\left(\wedge^{2} G^{*}\right)\right) \rightarrow \wedge^{2} T^{*} M .
$$

It is a not a vector bundle morphism but just a smooth fiberwise map. It maps the zero section to $\eta$, and its image is an open neighborhood of $\eta$ in the space of 2 -forms having the same rank as $\eta$. The map $\exp _{\eta}$ allows to parametrize deformations of $\eta$ inside $\operatorname{Pre} \operatorname{Sym}^{k}(M)$ by means of sections $(\mu, \sigma) \in \Gamma\left(K^{*} \otimes G^{*}\right) \oplus \Gamma\left(\wedge^{2} G^{*}\right) \cong \Omega_{\text {hor }}^{2}(M)$ which are sufficiently small in the $C^{0}$-sense and for which the 2 -form $\left(\exp _{\eta}\right)(\mu, \sigma)$ is a closed. 


\subsection{An $L_{\infty}$-algebra associated to a bivector field}

In this subsection we canonically associate an $L_{\infty}$-algebra to any bivector field $Z$ on a manifold $M$.

Definition 2.5. Let $Z$ be a bivector field on $M$. The Koszul bracket associated to $Z$ is the operation

$$
\begin{aligned}
& {[\cdot, \cdot]_{Z}: \Omega^{r}(M) \times \Omega^{s}(M) \rightarrow \Omega^{r+s-1}(M),} \\
& {[\alpha, \beta]_{Z}:=(-1)^{|\alpha|+1}\left(\mathcal{L}_{Z}(\alpha \wedge \beta)-\mathcal{L}_{Z}(\alpha) \wedge \beta-(-1)^{|\alpha|} \alpha \wedge \mathcal{L}_{Z}(\beta)\right) .}
\end{aligned}
$$

Here $\mathcal{L}_{Z}=\iota_{Z} \circ d-d \circ \iota_{Z}$, where $\iota_{Z}$ denotes contraction with $Z$ and $d$ is the de Rham differential. On 1-forms $\alpha$ and $\beta$, the Koszul bracket reads $[\alpha, \beta]_{Z}=\mathcal{L}_{Z^{\sharp} \alpha} \beta-\mathcal{L}_{Z^{\sharp} \beta} \alpha-d\langle Z, \alpha \wedge \beta\rangle$.

In general the Koszul bracket of $Z$ does not satisfy the graded Jacobi identity (it does only when $Z$ is a Poisson bivector-field). We will see in Proposition 2.7 that nevertheless there is a well-behaved algebraic structure associated to $Z$. To this aim, recall that a differential form $\alpha \in \Omega^{r}(M)$ induces by contraction a linear map

$$
\alpha^{\sharp}: T M \rightarrow \wedge^{r-1} T^{*} M, \quad v \mapsto \iota_{v} \alpha,
$$

and, following [2, Section 2.3], we extend this definition to a collection of forms $\alpha_{1}, \ldots, \alpha_{n}$ by setting

$$
\begin{aligned}
\alpha_{1}^{\sharp} \wedge \cdots \wedge \alpha_{n}^{\sharp}: & \wedge^{n} T M \rightarrow \wedge^{\left|\alpha_{1}\right|+\cdots+\left|\alpha_{n}\right|-n} T^{*} M, \\
v_{1} & \wedge \cdots \wedge v_{n} \mapsto \sum_{\sigma \in S_{n}}(-1)^{|\sigma|} \alpha_{1}^{\sharp}\left(v_{\sigma(1)}\right) \wedge \cdots \wedge \alpha_{n}^{\sharp}\left(v_{\sigma(n)}\right) .
\end{aligned}
$$

Definition 2.6. We define the trinary bracket $[\cdot, \cdot, \cdot]_{Z}: \Omega^{r}(M) \times \Omega^{s}(M) \times \Omega^{k}(M) \rightarrow \Omega^{r+s+k-3}(M)$ associated to the bivector field $Z$ to be

$$
[\alpha, \beta, \gamma]_{Z}:=\left(\alpha^{\sharp} \wedge \beta^{\sharp} \wedge \gamma^{\sharp}\right)\left(\frac{1}{2}[Z, Z]\right) .
$$

These brackets endow $\Omega(M)[2]$ with an $L_{\infty}[1]$-algebra structure, extending the results of Fiorenza and Manetti [5]. The following is [7, Proposition 3.5]:

Proposition 2.7 (the $L_{\infty}[1]$-algebra $\Omega(M)[2]$ ). Let $Z$ be a bivector field on $M$. The multilinear maps $\lambda_{1}, \lambda_{2}, \lambda_{3}$ on the graded vector space $\Omega(M)[2]$ given by

1) $\lambda_{1}$ the de Rham differential $d$,

2) $\lambda_{2}(\alpha[2] \odot \beta[2])=-\left(\mathcal{L}_{Z}(\alpha \wedge \beta)-\mathcal{L}_{Z}(\alpha) \wedge \beta-(-1)^{|\alpha|} \alpha \wedge \mathcal{L}_{Z}(\beta)\right)[2]=(-1)^{|\alpha|}\left([\alpha, \beta]_{Z}\right)[2]$, and

3) $\lambda_{3}(\alpha[2] \odot \beta[2] \odot \gamma[2])=(-1)^{|\beta|+1}\left(\alpha^{\sharp} \wedge \beta^{\sharp} \wedge \gamma^{\sharp}\left(\frac{1}{2}[Z, Z]\right)\right)[2]$,

define the structure of an $L_{\infty}[1]$-algebra on $\Omega(M)[2]$.

We now explain the geometric relevance of the $L_{\infty}[1]$-algebra $\left(\Omega(M)[2], \lambda_{1}, \lambda_{2}, \lambda_{3}\right)$. As for any $L_{\infty}[1]$-algebra, it comes with distinguished elements:

Definition 2.8. An element $\beta \in \Omega^{2}(M)$ is a Maurer-Cartan element of $\left(\Omega(M)[2], \lambda_{1}, \lambda_{2}, \lambda_{3}\right)$ if it satisfies the Maurer-Cartan equation

$$
d(\beta[2])+\frac{1}{2} \lambda_{2}(\beta[2] \odot \beta[2])+\frac{1}{6} \lambda_{3}(\beta[2] \odot \beta[2] \odot \beta[2])=0 .
$$

Recall that at the beginning of Section 2.2 we defined an open subset $\mathcal{I}_{Z} \subset \wedge^{2} T^{*} M$ and a map $F: \mathcal{I}_{Z} \rightarrow \wedge^{2} T^{*} M$. The following is [7, Corollary 3.9].

Corollary 2.9 (Maurer-Cartan elements of $\Omega(M)[2]$ ). There is an open subset $\mathcal{U} \subset \mathcal{I}_{Z}$, which contains the zero section of $\wedge^{2} T^{*} M$, such that a 2 -form $\beta \in \Gamma(\mathcal{U})$ is a Maurer-Cartan element of $\left(\Omega(M)[2], \lambda_{1}, \lambda_{2}, \lambda_{3}\right)$ if, and only if, the 2 -form $F(\beta)$ is closed.

In Section 3.4 we will show that for the open subset $\mathcal{U}$ one can choose the whole of $\mathcal{I}_{Z}$. 


\subsection{The Koszul $L_{\infty}$-algebra of a pre-symplectic manifold}

Let again $\eta$ be a pre-symplectic structure on a manifold $M$. Fix a subbundle $G \subset T M$ which is complementary to the kernel $K$ of $\eta$. Consider the bivector field $Z$ satisfying $Z^{\sharp}=-\left(\left.\eta\right|_{G} ^{\sharp}\right)^{-1}$. The following is [7, Theorem 3.17].

Theorem 2.10 (the Koszul $L_{\infty}[1]$-algebra). The $L_{\infty}[1]$-algebra structure on $\Omega(M)[2]$ associated to the bivector field $Z$, see Proposition 2.7, maps $\Omega_{\mathrm{hor}}(M)[2]$ to itself. The subcomplex $\Omega_{\text {hor }}(M)[2] \subset \Omega(M)[2]$ therefore inherits the structure of an $L_{\infty}[1]$-algebra, which we call the Koszul $L_{\infty}[1]$-algebra of $(M, \eta)$.

We denote by $\mathrm{MC}(\eta)$ the set of Maurer-Cartan elements of the Koszul $L_{\infty}$ [1]-algebra of $(M, \eta)$.

In view of the above theorem, the following result [7, Theorem 3.19] is an immediate consequence of Theorem 2.3 and Corollary 2.9.

Theorem 2.11 (Maurer-Cartan elements of the Koszul $L_{\infty}[1]$-algebra). Let $(M, \eta)$ be a presymplectic manifold. The choice of a complement $G$ to the kernel of $\eta$ determines a bivector field $Z$ by requiring $Z^{\sharp}=-\left(\left.\eta\right|_{G} ^{\sharp}\right)^{-1}$. Suppose $\beta$ is a 2 -form on $M$, which lies in $\mathcal{I}_{Z}$. The following statements are equivalent:

1. $\beta$ is a Maurer-Cartan element of the Koszul $L_{\infty}[1]$-algebra $\Omega_{\mathrm{hor}}(M)[2]$ of $(M, \eta)$, which was introduced in Theorem 2.10 .

2. The image of $\beta$ under the map $\exp _{\eta}$, which is introduced in Definition 2.2, is a presymplectic structure of the same rank as $\eta$.

The above Theorem 2.11 is the main result of [7], as it states that the Koszul $L_{\infty}$ [1]-algebra governs the deformations of the pre-symplectic structure $\eta$. More precisely: the fibrewise map $\exp _{\eta}$ as in (2.2), on the level of sections, restricts to a map

$$
\exp _{\eta}: \Gamma\left(\mathcal{I}_{Z}\right) \cap \mathrm{MC}(\eta) \rightarrow{\operatorname{Pre}-S_{y m}}^{k}(M)
$$

which is injective and whose image consists of the pre-symplectic structures of rank equal to the rank of $\eta$ and with kernel transverse to $G$.

\section{Dirac geometric interpretation}

In the remainder of this note we explain the geometric framework that underlies the results of Section 2 recalled from [7]. We recover naturally the statements made there and provide some alternative and more geometric proofs.

\subsection{Background on Dirac geometry}

We first review some notions from Dirac linear algebra. Let $V$ be a finite-dimensional, real vector space. We denote by $\mathbb{V}$ the direct sum $V \oplus V^{*}$ and by $\langle\cdot, \cdot\rangle$ the following non-degenerate pairing on $\mathbb{V}$ :

$$
\langle(v, \xi),(w, \chi)\rangle:=\xi(w)+\chi(v) .
$$

Definition 3.1. A subspace $W \subset \mathbb{V}$ is called Lagrangian if for all $w, w^{\prime} \in W$ we have $\left\langle w, w^{\prime}\right\rangle=0$ and $\operatorname{dim}(W)=\operatorname{dim}(V)$. Two subspaces $W$ and $\tilde{W} \subset \mathbb{V}$ are transverse, if $W \oplus \tilde{W}=\mathbb{V}$. 
Given an element $Z \in \wedge^{2} V$, we defined the linear map $Z^{\sharp}: V^{*} \rightarrow V$ in Section 2.2, and we can consider the Lagrangian subspace $\operatorname{graph}(Z):=\left\{\left(Z^{\sharp} \xi, \xi\right) \mid \xi \in V^{*}\right\} \subset \mathbb{V}$. Similarly, for $\beta \in \wedge^{2} V^{*}$ we define $\beta^{\sharp}: V \rightarrow V^{*}$ and consider $\operatorname{graph}(\beta)$.

Every $\beta \in \wedge^{2} V^{*}$ defines an orthogonal transformation $\mathfrak{t}_{\beta}$ of $(\mathbb{V},\langle\cdot, \cdot\rangle)$, by

$$
(v, \xi) \mapsto\left(v, \xi+\beta^{\sharp}(v)\right) .
$$

Similarly, every $Z \in \wedge^{2} V$ gives rise to an orthogonal transformation $\mathfrak{t}_{Z}$, which takes $(v, \xi)$ to $\left(v+Z^{\sharp}(\xi), \xi\right)$. In particular, elements of $\wedge^{2} V^{*}$ and $\wedge^{2} V$ act on the set of Lagrangian subspaces of $\mathbb{V}$.

Remark 3.2. Suppose $L, R$ are transverse Lagrangian subspaces of $\mathbb{V}$. There is a canonical isomorphism

$$
R \cong L^{*},\left.\quad r \mapsto\langle r, \cdot\rangle\right|_{L}
$$

Since $R$ is transverse to $L$, any subspace of $\mathbb{V}$ transverse to $R$ is the graph of a linear map $L \rightarrow R$. Any Lagrangian subspace transverse to $R$ is the graph of a linear map $L \rightarrow R$ such that, composing with the canonical isomorphism above, we obtain a skew-symmetric linear map $L \rightarrow L^{*}$ (i.e., the sharp map associated to an element of $\wedge^{2} L^{*}$ ).

Let us now briefly recall the basic constituencies of Dirac geometry. Consider the generalized tangent bundle $\mathbb{T} M=T M \oplus T^{*} M$. It comes equipped with a non-degenerate pairing

$$
\langle(X, \alpha),(Y, \beta)\rangle:=\alpha(Y)+\beta(X)
$$

and the Dorfman bracket

$$
\llbracket(X, \alpha),(Y, \beta) \rrbracket=\left([X, Y], \mathcal{L}_{X} \beta-\iota_{Y} d \alpha\right) .
$$

Together with the projection to $T M$, this makes $\mathbb{T} M$ into an example of Courant algebroid.

Definition 3.3. An almost Dirac structure on $M$ is a Lagrangian subbundle $L \subset(\mathbb{T} M,\langle\cdot, \cdot\rangle)$. A Dirac structure is an almost Dirac structure whose space of sections is closed with respect to the Dorfman bracket $\llbracket \cdot, \cdot \rrbracket$.

Remark 3.4. Let $L, R$ be transverse Dirac structures on $M$. As seen in Remark 3.2, almost Dirac structures transverse to $R$ are in bijection with elements of $\Gamma\left(\wedge^{2} L^{*}\right)$. We now recall a result of Liu-Weinstein-Xu [4] establishing when such an almost Dirac structure is Dirac. Recall that every Dirac structure, with the restricted Dorfman bracket and anchor, is a Lie algebroid. Since $L$ is a Lie algebroid, it induces a differential $d_{L}$ on $\Gamma\left(\wedge L^{*}\right)$. Further ${ }^{1}$, since $L^{*} \cong R$ is a Lie algebroid, it induces a graded Lie bracket $[\cdot, \cdot]_{L^{*}}$ on $\Gamma\left(\wedge L^{*}\right)[1]$. Together with $d_{L}$ and $[\cdot, \cdot]_{L^{*}}$, the graded vector space $\Gamma\left(\wedge L^{*}\right)[1]$ becomes a differential graded Lie algebra. The main result of [4] is: for all $\varepsilon \in \Gamma\left(\wedge^{2} L^{*}\right)$, the graph $L_{\varepsilon}=\left\{v+\iota_{v} \varepsilon: v \in L\right\}$ is a Dirac structure iff $\varepsilon$ satisfies the Maurer-Cartan equation, that is

$$
d_{L} \varepsilon+\frac{1}{2}[\varepsilon, \varepsilon]_{L^{*}}=0 .
$$

\footnotetext{
${ }^{1}$ The Lie algebroid structures on $L$ and $L^{*}$ are compatible in the sense that the pair $\left(L, L^{*}\right)$ forms a Lie bialgebroid.
} 


\subsection{Deformations of pre-symplectic structures: the point of view of Dirac geometry}

In this subsection we cast the deformations of pre-symplectic forms in the framework of Dirac geometry.

Let $\eta$ be a pre-symplectic form on $M$, with kernel $K$. The natural way to parametrize deformations of $\eta$ is by 2 -forms $\alpha$ such that $\eta+\alpha$ is again pre-symplectic, but this parametrization has a serious flaw: the space of such $\alpha$ 's does not have a natural vector space structure, due to the constant rank condition. Taking the point of view of Dirac geometry, the above approach to parametrize the deformations of $\eta$ amounts to deforming the Dirac structure graph $(\eta)$ using $\{0\} \oplus T^{*} M$ as a complement.

A better way to parametrize the deformations of $\eta$ in terms of Dirac geometry works as follows. Let us first choose a complement $G$ to $K$. Then

$$
G \oplus K^{*}
$$

is a complement ${ }^{2}$ of $\operatorname{graph}(\eta)$. We can now use $G \oplus K^{*}-\operatorname{instead}$ of $\{0\} \oplus T^{*} M$ - to parametrize deformations of the Dirac structure graph $(\eta)$. This choice of complement has the advantage of linearizing the constant rank condition, as we show in Proposition 3.7 below. (Notice that when $\eta$ is symplectic, the new complement is just $T M$, hence we are deforming $\eta$ by viewing it as a Poisson structure, just as in [7, Section 1.3].)

We first state two lemmas about the effect of applying the orthogonal transformation $\mathfrak{t}_{-\eta}$ of $T M \oplus T^{*} M$, given by $(v, \xi) \mapsto\left(v, \xi-\eta^{\sharp}(v)\right)$.

Lemma 3.5. Denote by $Z \in \Gamma\left(\wedge^{2} G\right)$ the bivector field such that $Z^{\sharp}$ is the inverse of $-\left(\left.\eta\right|_{G}\right)^{\sharp}$. Then $\mathfrak{t}_{-\eta}$ maps $G \oplus K^{*}$ to $\operatorname{graph}(Z)$.

Proof. $\mathfrak{t}_{\eta}(\operatorname{graph}(Z))=\left\{\left(Z^{\sharp} \xi,\left.\xi\right|_{K}\right): \xi \in T^{*} M\right\}=G \oplus K^{*}$.

Lagrangian subbundles nearby $\operatorname{graph}(\eta)$ can be written, for some $\bar{\beta} \in \Gamma\left(\wedge^{2}(\operatorname{graph}(\eta))^{*}\right)$, as the graph of the map

$$
\bar{\beta}^{\sharp}: \operatorname{graph}(\eta) \rightarrow(\operatorname{graph}(\eta))^{*} \cong G \oplus K^{*},
$$

by Remark 3.2. We denote this graph as $\Phi_{G \oplus K^{*}}(\bar{\beta})$. Moreover, let $\beta \in \Omega^{2}(M)$ be the 2 -form corresponding to $\bar{\beta}$ under the isomorphism $\operatorname{graph}(\eta) \cong T M, v+\iota_{v} \eta \mapsto v$ and denote by $\Phi_{Z}(\beta)$ the graph of the map $\beta^{\sharp}: T M \rightarrow T^{*} M \cong \operatorname{graph}(Z)$.

Lemma 3.6. $\mathfrak{t}_{-\eta}$ maps $\Phi_{G \oplus K^{*}}(\bar{\beta})$ to $\Phi_{Z}(\beta)$.

Proof. $\mathfrak{t}_{-\eta}$ preserves the pairing on $T M \oplus T^{*} M$, clearly maps $\operatorname{graph}(\eta)$ to $T M$, and maps $G \oplus K^{*}$ to $\operatorname{graph}(Z)$ by Lemma 3.5. Therefore the statement follows by functoriality.

Now we can explain why the choice of $G \oplus K^{*}$ as a complement is a good one to describe pre-symplectic deformations.

Proposition 3.7. Let $\bar{\beta} \in \Gamma\left(\wedge^{2}(\operatorname{graph}(\eta))^{*}\right)$.

(i) The rank of

$$
\Phi_{G \oplus K^{*}}(\bar{\beta}) \cap T M
$$

equals the rank of

$$
\left\{v \in K: \iota_{v} \beta \in G^{*}\right\} .
$$

\footnotetext{
${ }^{2}$ Indeed, for every $v \in T M$ we have $\iota_{v} \eta \in K^{\circ}=G^{*}$, so requiring that $\iota_{v} \eta$ lies in $K^{*}$ implies $\iota_{v} \eta=0$. This means that $v \in K$, so requiring that $v$ lies in $G$ implies $v=0$.
} 
(ii) Assume that $\Phi_{G \oplus K^{*}}(\bar{\beta})$ is the graph of a 2-form. Then the rank of this 2-form equals $\operatorname{rank}(\eta)$ iff $\beta$ lies in the vector space $\Omega_{\mathrm{hor}}^{2}(M)$ of horizontal 2-forms.

Proof. (i) Applying the transformation $\mathfrak{t}_{-Z} \circ \mathfrak{t}_{-\eta}$ to $\Phi_{G \oplus K^{*}}(\bar{\beta})$, by Lemma 3.6 we obtain $\mathfrak{t}_{-Z}\left(\Phi_{Z}(\beta)\right)=\operatorname{graph}(\beta)$. Applying it to $T M$ we obtain $\left\{\left(v+Z^{\sharp} \iota_{v} \eta,-\iota_{v} \eta\right) \mid v \in T M\right\}=K \oplus G^{*}$.

Hence applying the transformation to the intersection (3.1) we obtain

$$
\operatorname{graph}(\beta) \cap\left(K \oplus G^{*}\right)
$$

which is isomorphic to (3.2).

(ii) Denote by $\eta^{\prime}$ the 2 -form whose graph is $\Phi_{G \oplus K^{*}}(\bar{\beta})$. The kernel of $\eta^{\prime}$ is given by (3.1), and the assertion follows immediately from (i). Recall that the vector space $\Omega_{\text {hor }}^{2}(M)$ of horizontal 2 -forms was defined in Section 2.1, as the space of 2-forms that vanish on $\wedge^{2} K$.

Remark 3.8. Since $\mathfrak{t}_{-\eta}$ is actually an automorphism of the standard Courant algebroid $T M \oplus$ $T^{*} M$, the following two deformation problems of Dirac structures are equivalent:

- deformations of graph $(\eta)$, using the complement $G \oplus K^{*}$,

- deformations of $T M$, using the complement $\operatorname{graph}(Z)$.

The latter deformation problem is easier to handle, and the $L_{\infty}[1]$-algebra structure governing it will be recovered in Section 3.4.

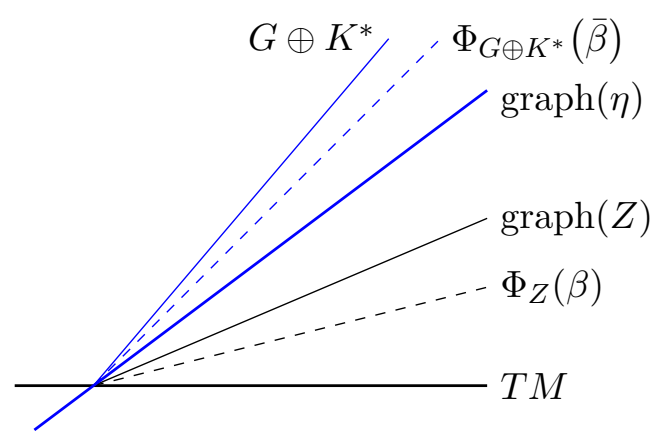

Figure 1. The Dirac structures graph $(\eta)$ and $T M$, together with the complementary Lagrangian subbundles we use to deform them.

\subsection{Dirac-geometric interpretation of Section 2.2}

Using Dirac linear algebra, we explain and re-prove the results recalled in Section 2.2.

\subsubsection{Revisiting the map $F$ from formula (2.1)}

Let $V$ be a finite-dimensional, real vector space. We fix a bivector $Z \in \wedge^{2} V$. Recall that $\mathcal{I}_{Z}$ consists of elements $\beta \in \wedge^{2} V^{*}$ such that id $+Z^{\sharp} \beta^{\sharp}$ is invertible. In formula (2.1), we defined the $\operatorname{map} F: \mathcal{I}_{Z} \rightarrow \wedge^{2} V^{*}$ given by

$$
(F(\beta))^{\sharp}=\beta^{\sharp}\left(\mathrm{id}+Z^{\sharp} \beta^{\sharp}\right)^{-1} \text {. }
$$

The following lemma provides a geometric explanation of the map $F$. 
Lemma 3.9. Fix $Z \in \wedge^{2} V$.

(i) Taking graphs with respect to the decompositions $\mathbb{V}=V \oplus V^{*}$ resp. $\mathbb{V}=V \oplus \operatorname{graph}(Z)$, yields bijections

$$
\begin{aligned}
\Phi_{0}: \wedge^{2} V^{*} & \left.\cong \text { Lagrangian subspace of } \mathbb{V} \text { transverse to } V^{*}\right\}, \\
\alpha & \mapsto\left\{\left(v, \iota_{v} \alpha\right) \mid v \in V\right\}, \\
\Phi_{Z}: \wedge^{2} V^{*} & \cong\{\text { Lagrangian subspace of } \mathbb{V} \text { transverse to } \operatorname{graph}(Z)\}, \\
\beta & \mapsto\left\{\left(v+Z^{\sharp}\left(\iota_{v} \beta\right), \iota_{v} \beta\right) \mid v \in V\right\} .
\end{aligned}
$$

(ii) Given $\beta \in \wedge^{2} V^{*}$, the Lagrangian subspace $\Phi_{Z}(\beta)$ is transverse to $V^{*} \subset \mathbb{V}$ if, and only if $\beta \in \mathcal{I}_{Z}$.

(iii) The map

$$
\Phi_{0}^{-1} \circ \Phi_{Z}: \mathcal{I}_{Z} \rightarrow \wedge^{2} V^{*}
$$

is well-defined and coincides with $F$.

In particular, the map $F$ is characterized by the property that

$$
\operatorname{graph}(F(\beta))=\Phi_{Z}(\beta)
$$

for all $\beta \in \mathcal{I}_{Z}$. In other words, $F(\beta)$ is obtained taking the graph of $\beta$ w.r.t. the splitting $\mathbb{V}=V \oplus \operatorname{graph}(Z)$.

Proof. (i) According to Remark 3.2, any Lagrangian subspace transverse to $V^{*}$ is the graph of a skew-symmetric linear map $V \rightarrow V^{*}$, and therefore can be written as $\left\{\left(v, \iota_{v} \alpha\right) \mid v \in V\right\}$ for some $\alpha \in \wedge^{2} V^{*}$. Similarly, $\operatorname{graph}(Z)$ is transverse to $V$ and the induced isomorphism $\operatorname{graph}(Z) \cong V^{*}$ is just $\left(Z^{\sharp}(\xi), \xi\right) \mapsto \xi$. Hence any Lagrangian subspace transverse to graph $(Z)$ can be written as $\left\{(v, 0)+\left(Z^{\sharp}\left(\iota_{v} \beta\right), \iota_{v} \beta\right) \mid v \in V\right\}$ for some $\beta \in \wedge^{2} V^{*}$.

(ii) The expression for $\Phi_{Z}(\beta)$ in item (i) shows that $\Phi_{Z}(\beta) \cap V^{*}=\left\{\left(0, \iota_{v} \beta\right) \mid v \in V, v+\right.$ $\left.Z^{\sharp}\left(\iota_{v} \beta\right)=0\right\}$. This intersection is trivial iff $\operatorname{ker}\left(\mathrm{id}+Z^{\sharp} \beta^{\sharp}\right) \subseteq \operatorname{ker}\left(\beta^{\sharp}\right)$. In turn, this condition is equivalent to (id $+Z^{\sharp} \beta^{\sharp}$ ) being injective, and thus invertible.

(iii) Finally, if id $+Z^{\sharp} \beta^{\sharp}$ is invertible, $\Phi_{Z}(\beta)$ is transverse to $V^{*}$ by item (ii). By item (i) the element $\Phi_{0}^{-1}\left(\Phi_{Z}(\beta)\right)$ is well-defined. In concrete terms, it is given by $\alpha \in \wedge^{2} V^{*}$ such that for all $v \in V$, there is $w \in V$ for which

$$
\left(v+Z^{\sharp} \beta^{\sharp}(v), \beta^{\sharp}(v)\right)=\left(w, \alpha^{\sharp}(w)\right)
$$

holds. Equivalently, this means that $\alpha^{\sharp}\left(\mathrm{id}+Z^{\sharp} \beta^{\sharp}\right)(v)=\beta^{\sharp}(v)$ for all $v \in V$. This shows that $\Phi_{0}^{-1} \circ \Phi_{Z}$ agrees with $F$.

\subsubsection{Revisiting Theorem 2.3 (parametrizing constant rank forms)}

Now let $\eta \in \wedge^{2} V^{*}$ be of rank $k$, fix a complement $G$ to $K:=\operatorname{ker}(\eta)$, and denote by $Z \in \wedge^{2} G$ the bivector determined by $Z^{\sharp}=-\left(\left.\eta\right|_{G} ^{\sharp}\right)^{-1}$. In Section 3.2 we considered deformations of the Dirac structure graph $(\eta)$ using $G \oplus K^{*}$ as a complement. They are graphs of 2 -forms given by the Dirac exponential map $\exp _{\eta}$ (see Definition 2.2). More precisely:

Lemma 3.10. For all $\beta \in \mathcal{I}_{Z}$ we have

$$
\operatorname{graph}\left(\exp _{\eta}(\beta)\right)=\Phi_{G \oplus K^{*}}(\bar{\beta}) .
$$


Proof. We have graph $\left(\exp _{\eta}(\beta)\right)=\mathfrak{t}_{\eta}\left(\Phi_{Z}(\beta)\right)=\Phi_{G \oplus K^{*}}(\bar{\beta})$, where the first equality holds by equation (3.3) and the second by Lemma 3.6.

Using this we recover Theorem 2.3, in particular item (i) stating that $\exp _{\eta}(\beta)$ has rank equal to $k=\operatorname{dim}(K)$ iff $\beta$ is horizontal.

Alternative proof of Theorem 2.3. (i) Apply Proposition 3.7(ii) together with equation (3.4).

(ii) We only prove the statement about the kernel of $\exp _{\eta}(\beta)$. Write $\beta=(\mu, \sigma)$. By the proof of Proposition 3.7(i), the intersection of the subspace (3.4) with $V$ is $\left(\mathfrak{t}_{\eta} \circ \mathfrak{t}_{Z}\right)\left(\operatorname{graph}(\beta) \cap\left(K \oplus G^{*}\right)\right)$, which is precisely the image of $K$ under id $+Z^{\sharp} \mu^{\sharp}$.

(iii) By Lemma 3.9(ii), the map $\Phi_{Z}$ provides a bijection between $\mathcal{I}_{Z}$ and Lagrangian subspaces transverse to graph $(Z)$ and to $V^{*}$. Hence $\mathfrak{t}_{\eta} \circ \Phi_{Z}$ provides a bijection between $\mathcal{I}_{Z}$ and Lagrangian subspaces transverse to $\mathfrak{t}_{\eta}(\operatorname{graph}(Z))=G \oplus K^{*}$ (see Lemma 3.5) and to $V^{*}$. The latter are exactly the graphs of elements $\eta^{\prime} \in \wedge^{2} V^{*}$ so that the $\left.\eta^{\prime}\right|_{\wedge^{2} G}$ is non-degenerate. Hence, by the proof of Lemma 3.10, $\exp _{\eta}$ provides a bijection between $\mathcal{I}_{Z}$ and such $\eta^{\prime}$. We conclude using (i).

\subsection{Dirac-geometric interpretation of Section 2.3}

Using Dirac geometry and adapting results from [2], we explain and re-prove the results recalled in Section 2.3. Fix a bivector field $Z$ on $M$.

\subsubsection{Revisiting Proposition 2.7 (the $L_{\infty}[1]$-algebra $\Omega(M)[2]$ )}

In Proposition 2.7, the $L_{\infty}[1]$-algebra $\left(\Omega(M)[2], \lambda_{1}, \lambda_{2}, \lambda_{3}\right)$ was constructed out of a bivector field $Z$. It can be recovered using Dirac geometry - or more precisely, the deformation theory of Dirac structures - as a special case of the construction from [2, Section 2.2].

Proposition 3.11. Let $L$ be a Dirac structure and $R$ a complementary almost Dirac structure, i.e., we have a vector bundle decomposition $L \oplus R=\mathbb{T} M$. Then $\Gamma\left(\wedge L^{*}\right)[2]$ has an induced $L_{\infty}[1]$-algebra structure, whose only non-trivial multibrackets are $\mu_{1}, \mu_{2}, \mu_{3}$ given as follows:

1) $\mu_{1}$ is the differential $d_{L}$ associated to the Lie algebroid $L$,

2) $\mu_{2}(\alpha[2] \odot \beta[2])=-(-1)^{|\alpha|}[\alpha, \beta]_{L^{*}}[2]$, where $[\cdot, \cdot]_{L^{*}}:=\operatorname{pr}_{R}(\llbracket \cdot, \cdot \rrbracket)$ denotes the (extension of) the bracket of the almost Lie algebroid $R \cong L^{*}$,

3) $\mu_{3}(\alpha[2] \odot \beta[2] \odot \gamma[2])=(-1)^{|\beta|}\left(\alpha^{\sharp} \wedge \beta^{\sharp} \wedge \gamma^{\sharp}\right) \psi[2]$, where $\psi \in \Gamma\left(\wedge^{3} L\right)$ is given by $\Gamma\left(\wedge^{3} L^{*}\right) \rightarrow$ $\mathcal{C}^{\infty}(M), \xi_{1} \wedge \xi_{2} \wedge \xi_{3} \mapsto\left\langle\operatorname{pr}_{L}\left(\llbracket \xi_{1}, \xi_{2} \rrbracket\right), \xi_{3}\right\rangle$, where we made use of the identification $R \cong L^{*}$.

More generally, Proposition 3.11 holds if replacing $\mathbb{T} M$ by any Courant algebroid.

Proof. The proof is a minor adaptation of the first part of the proof of [2, Lemma 2.6], setting $\varphi=0$ there. We recall briefly the idea of the latter. By [6] there is a natural description of the Courant algebroid structure on $\mathbb{T} M$ in terms of graded geometry. One can use it to apply Voronov's higher derived brackets construction (see [8,9]) and obtain an $L_{\infty}[1]$-algebra structure on $\Gamma\left(\wedge L^{*}\right)[2]$. The multibrackets obtained are the ones in the statement of the lemma, as one checks using [6] and via computations in local coordinates.

Alternative proof of Proposition 2.7. Let $Z$ be a bivector field on $M$. We apply Proposition 3.11 for the case $L=T M$ and $R=\operatorname{graph}(Z)$. In this case $d_{L}$ is the de Rham differential, and the bracket on $R$ is given by the formula for the Koszul bracket. One checks that $\psi$ is the trivector field $-\frac{1}{2}[Z, Z]$, using [7, Lemma 1.6]. Hence the $L_{\infty}$ [1]-brackets on $\Omega(M)[2]$ given by Proposition 3.11 are $\mu_{1}=\lambda_{1}, \mu_{2}=-\lambda_{2}$ and $\mu_{3}=\lambda_{3}$. Applying the automorphism -id to $\Omega(M)[2]$ yields Proposition 2.7. 


\subsubsection{Revisiting Corollary 2.9 (Maurer-Cartan elements of $\Omega(M)[2]$ )}

We now turn to Maurer-Cartan elements. In Lemma 3.9(i), we gave a parametrization of all almost Dirac structures that are transverse to $\operatorname{graph}(Z)$ in terms of 2 -forms $\beta$ on $M$. This parametrization is given by

$$
\beta \mapsto \Phi_{Z}(\beta)=\left\{\left(v+Z^{\sharp}\left(\iota_{v} \beta\right), \iota_{v} \beta\right) \mid v \in T M\right\} .
$$

We present the second part of [2, Lemma 2.6], which is an extension of the work by LiuWeinstein-Xu recalled in Remark 3.4.

Proposition 3.12. Let $L$ be given a Dirac structure and $R$ a complementary almost Dirac structure. An element $\sigma \in \Gamma\left(\wedge^{2} L^{*}\right)[2]$ is a Maurer-Cartan element of the $L_{\infty}[1]$-algebra structure given in Proposition 3.11 iff the graph

$$
\Gamma_{\sigma}:=\left\{\left(X-\iota_{X} \sigma\right): X \in L\right\} \subset L \oplus R
$$

is a Dirac structure. (The above inclusion makes use of the identification $R \cong L^{*}$.)

Corollary 2.9 states that for $\beta \in \Omega^{2}(M)$ taking values in some sufficiently small neighborhood $\mathcal{U}$ of the zero section in $\wedge^{2} T^{*} M$ - in particular taking values in $\mathcal{I}_{Z}$, i.e., id $+Z^{\sharp} \beta^{\sharp}$ is invertible, $-\beta$ is a Maurer-Cartan element of $\left(\Omega(M)[2], \lambda_{1}, \lambda_{2}, \lambda_{3}\right)$ iff $F(\beta)$ is closed. We now provide an alternative proof of this result, which also shows that one can choose $\mathcal{U}$ to equal $\mathcal{I}_{Z}$.

Alternative proof of Corollary 2.9. For any $\beta \in \Omega^{2}(M)$, being a Maurer-Cartan element of the $L_{\infty}$ [1]-algebra $\left(\Omega(M)[2], \lambda_{1}, \lambda_{2}, \lambda_{3}\right)$ is equivalent to $\Phi_{Z}(\beta)$ being a Dirac structure. This follows from applying Proposition 3.12 to the Dirac structure $L=T M$ and to the almost Dirac structure $R=\operatorname{graph}(Z)$, noticing that $\Gamma_{-\beta}=\left\{\left(v+Z^{\sharp}\left(\iota_{v} \beta\right), \iota_{v} \beta\right) \mid v \in T M\right\}=\Phi_{Z}(\beta)$. When $\beta \in \Gamma\left(\mathcal{I}_{Z}\right)$, we know that $\Phi_{Z}(\beta)$ can be written as the graph of the 2 -form $F(\beta)$, by equation (3.3). Now use the fact that the graph of a 2 -form is a Dirac structure if, and only if, the 2 -form is closed.

Remark 3.13. In this subsection we recovered the $L_{\infty}$ [1]-algebra $\Omega(M)[2]$ of Proposition 2.7 as the $L_{\infty}[1]$-algebra governing deformations of the Dirac structure $T M$ taking $\operatorname{graph}(Z)$ as a complement. By Remark 3.8, this deformation problem is equivalent to the deformations of the Dirac structure $\operatorname{graph}(\eta)$ taking $G \oplus K^{*}$ as the complement. This explains why the $L_{\infty}[1]$-algebra $\Omega(M)[2]$ governs the latter deformation problem, and therefore is relevant for the deformations of pre-symplectic structures.

\subsection{Dirac-geometric interpretation of Section 2.4}

Theorem 2.10 can be deduced from a general statement about (almost) Dirac structures, however doing so amounts essentially to the same computations that were needed for the proof given in [7]. We include this general statement for the sake of completeness.

Proposition 3.14. In the setting of Proposition 3.11, let $K$ be a subbundle of $L$ and define $\Gamma_{\text {hor }}\left(\wedge L^{*}\right)$ as the kernel of the restriction map $\Gamma\left(\wedge L^{*}\right) \rightarrow \Gamma\left(\wedge K^{*}\right)$. Then the multibrackets $\mu_{1}$, $\mu_{2}, \mu_{3}$ preserve $\Gamma_{\text {hor }}\left(\wedge L^{*}\right)[2]$ iff $K$ satisfies the following:

- $K$ is a Lie subalgebroid of $L$,

- $\left\langle\llbracket \xi_{1}, \xi_{2} \rrbracket, K+K^{\circ}\right\rangle=0$ for all $\xi_{1}, \xi_{2} \in \Gamma\left(K^{\circ}\right)$, where we use the identification $K^{\circ} \subset L^{*} \cong R$ and $\llbracket \cdot, \cdot \rrbracket$ denotes the Dorfman bracket. 
Proof. We will use the fact that $\mu_{1}, \mu_{2}, \mu_{3}$ are derivations w.r.t. the wedge product in each entry. The Lie algebroid differential $d_{L}$ preserves $\Gamma_{\text {hor }}\left(\wedge L^{*}\right)$ iff the subbundle $K$ is involutive. The bracket $[\cdot, \cdot]_{L^{*}}$ preserves $\Gamma_{\text {hor }}\left(\wedge L^{*}\right)$ iff $\left\langle\llbracket \xi_{1}, \xi_{2} \rrbracket, K\right\rangle=0$ for all $\xi_{1}, \xi_{2} \in \Gamma\left(K^{\circ}\right)$. The trinary bracket $\mu_{3}$ preserves $\Gamma_{\text {hor }}\left(\wedge L^{*}\right)$ iff $\mu_{3}\left(\xi_{1}, \xi_{2}, \xi_{3}\right)=0$ for all $\xi_{i} \in \Gamma\left(K^{\circ}\right)$, which in turn is equivalent to $\left\langle\llbracket \xi_{1}, \xi_{2} \rrbracket, \xi_{3}\right\rangle=0$.

Finally, as mentioned earlier, Theorem 2.11 follows immediately from the other results presented.

\section{Acknowledgements}

We thank Stephane Geudens for comments on a draft of this note. M.Z. acknowledges partial support by IAP Dygest, the long term structural funding - Methusalem grant of the Flemish Government, the FWO under EOS project G0H4518N, the FWO research project G083118N (Belgium).

\section{References}

[1] Fiorenza D., Manetti M., Formality of Koszul brackets and deformations of holomorphic Poisson manifolds, Homology Homotopy Appl. 14 (2012), 63-75, arXiv:1109.4309.

[2] Frégier Y., Zambon M., Simultaneous deformations and Poisson geometry, Compos. Math. 151 (2015), 1763-1790, arXiv:1202.2896.

[3] Gualtieri M., Matviichuk M., Scott G., Deformation of Dirac structures via $L_{\infty}$ algebras, Int. Math. Res. Not., to appear, arXiv:1702.08837.

[4] Liu Z.-J., Weinstein A., Xu P., Manin triples for Lie bialgebroids, J. Differential Geom. 45 (1997), 547-574, dg-ga/9508013.

[5] Manetti M., On some formality criteria for DG-Lie algebras, J. Algebra 438 (2015), 90-118, arXiv:1310.3048.

[6] Roytenberg D., Courant algebroids, derived brackets and even symplectic supermanifolds, Ph.D. Thesis, University of California at Berkeley, math.DG/9910078.

[7] Schätz F., Zambon M., Deformations of pre-symplectic structures and the Koszul $L_{\infty}$-algebra, Int. Math. Res. Not., to appear, arXiv:1703.00290.

[8] Voronov T., Higher derived brackets and homotopy algebras, J. Pure Appl. Algebra 202 (2005), 133-153, math.QA/0304038.

[9] Voronov T., Higher derived brackets for arbitrary derivations, in Travaux mathématiques. Fasc. XVI, Trav. Math., Vol. 16, University of Luxembourg, Luxembourg, 2005, 163-186, math.QA/0412202. 